D. Preiss, Department of Mathematics, University College London, London WC1E 6BT, United Kingdom. e-mail: dp@math.ucl.ac.uk

L. Zajiček, Department of Math. Anal., Charles University, Sokolovská 83, 18600 Prague 8, Czech Republic. e-mail: zajicek@karlin.mff.cuni.cz

\title{
SIGMA-POROUS SETS IN PRODUCTS OF METRIC SPACES AND SIGMA-DIRECTIONALLY POROUS SETS IN BANACH SPACES
}

\begin{abstract}
We show that no reasonable classical form of "Fubini type theorems" can hold for the $\sigma$-ideal of $\sigma$-porous sets in products of metric spaces (even in the plane). Then we prove that a "Fubini type theorem" in a weak decomposition form remains true also for this $\sigma$-ideal, and we illustrate how this fact may be applied to the study of the behavior of measures on small sets in product spaces. We also prove an analogical decomposition theorem for $\sigma$-directionally porous sets in Banach spaces; such sets arise naturally as exceptional sets in some questions concerning differentiability properties of Lipschitz functions on Banach spaces.
\end{abstract}

\section{Introduction}

The Fubini theorem in $\mathbb{R}^{2}$ immediately implies the following statements.

(a) If $M \subset \mathbb{R}^{2}$ is a Lebesgue measurable set and for all $x \in \mathbb{R}$, except a Lebesgue null set, the section $M_{x}$ is Lebesgue null, then $M$ is a Lebesgue null set.

(b) If $M \subset \mathbb{R}^{2}$ is of Lebesgue measure zero, then, for all $x \in \mathbb{R}$ except a (onedimensional) Lebesgue null set, the section $M_{x}=\{y \in \mathbb{R}:(x, y) \in M\}$ is Lebesgue null.

Key Words: Porous sets, directionally porous set, Fubini theorem

Mathematical Reviews subject classification: 28A05, 26B05, 46G99

Received by the editors November 6, 1997

* Supported by grants GAČR 201/97/1161 and GAUK 190/1996 
(c) If $M \subset \mathbb{R}^{2}$ is a Borel set of Lebesgue measure zero, then $M$ can be written as $M=A \cup B$, where $A, B \subset \mathbb{R}^{2}$ are Borel sets such that, for all $x \in \mathbb{R}$ the section $A_{x}=\{y \in \mathbb{R}:(x, y) \in A\}$ is Lebesgue null and for all $y \in \mathbb{R}$ the section $B^{y}=\{x \in \mathbb{R}:(x, y) \in B\}$ is Lebesgue null.

The question whether Fubini type theorems; i.e., analogies of the statements (a) and (b), hold also for sets small in the sense of Baire category is answered positively by the well-known Kuratowski-Ulam theorem (cf. [O]). The statement (c) seems to be mentioned only rarely, probably because it is a very easy consequence of (b); we are aware only of its use in the definition of sets null in the sense of Aronszajn (see [A]). It is therefore surprising that for the $\sigma$-ideal of $\sigma$-porous sets the analogy of (c) appears as the only correct generalization of the Fubini theorem; and that this seemingly weak generalization has also interesting applications.

Let us first consider the classical formulations of Fubini type theorems. It is well known that the analogy of (a) does not hold for the $\sigma$-ideal of $\sigma$-porous sets. In fact, Foran in $[F]$ constructed a continuous function $f: \mathbb{R} \rightarrow \mathbb{R}$ which has a non- $\sigma$-porous graph. Thus the " $\sigma$-porous analogue" of (a) is invalid even for some closed sets $M$. On the other hand, in the (very) special case when $M$ is an analytic set of the form $M=A \times B$ the analogue of (a) holds. This follows from [Z4], where it is shown that $A \times B$ is non- $\sigma$-porous whenever $A, B$ are $G_{\delta}$ non- $\sigma$-porous subsets of $\mathbb{R}$ and the fact that each analytic non- $\sigma$-porous subset of $\mathbb{R}$ contains a closed non- $\sigma$-porous set. The last result was obtained independently by different methods by J. Pelant and M. Zelený; the proofs are still unpublished.

In the second section of our article, we give a counterexample showing that no statement directly analogous to (b) can hold for the $\sigma$-ideal of $\sigma$-porous sets. We even show that there exists a set $M \subset \mathbb{R}^{2}$ which is porous in a rather strict sense and $\mathbb{R} \backslash M_{x}$ is Lebesgue null for each $x \in \mathbb{R}$ except a first category set.

In the third section we prove our $\sigma$-porous analogy of the weak Fubini theorem (c) saying that if $X, Y$ are metric spaces and $M \subset X \times Y$ is a Borel $\sigma$-porous set, then there exists a decomposition $M=A \cup B$, where $A, B$ are Borel and all sections $A_{x}$ and $B^{y}$ are $\sigma$-porous. In fact, our decomposition theorem (Theorem 3.8) shows considerably more. $A$ and $B$ are in certain sense $\sigma$-porous "in the direction of $Y$ and $X$ ", respectively.

As a consequence, we obtain the following fact (see Proposition 3.10).

If $\mu$ and $\nu$ are Radon measures on separable metric spaces $X$ and $Y$, respectively, which are "absolutely continuous" w.r.t. $\sigma$-porous sets, then the Radon product measure $\mu \otimes \nu$ is "absolutely continuous" w.r.t. $\sigma$-porous sets as well. 
We also obtain decomposition theorems similar to the one described above for $\sigma$-directionally porous sets in separable Banach spaces. It should be noted that the class of $\sigma$-directionally porous sets arises quite naturally in some questions concerning differentiability properties of Lipschitz functions on Banach spaces. Here we mention only one sample result from [PZ].

If $f$ is a Lipschitz function defined on a separable Banach space $X$, then, for all points $a \in X$ except those which belong to a $\sigma$-directionally porous set, the function $g(v):=\lim \sup _{h \rightarrow 0+} \frac{f(a+h v)-f(a)}{h}$ is convex.

We adopt the following notation.

In a metric space, the open ball with center $x$ and radius $r$ will be denoted by $B(x, r)$. The distance of two sets $A, B$ is denoted by $\operatorname{dist}(A, B)$. The closure and the interior of a set $A$ are denoted by $\bar{A}$ and $\operatorname{int} A$, respectively. The Lebesgue measure on $\mathbb{R}$ is denoted by $\lambda$. The linear span of a subset $M$ of a linear space is denoted by $\operatorname{span} M$.

Now we recall definitions of some porosity notions and present basic relevant comments.

Definition 1.1. Let $(X, \rho)$ be a metric space, $M \subset X$ and $a \in X$. Then we say that

(i) $M$ is porous at $a$ if there exists $c>0$ such that for each $\varepsilon>0$ there exists $b \in X$ and $r>c \rho(a, b)$ such that $\rho(a, b)<\varepsilon$ and $M \cap B(b, r)=\emptyset$.

(ii) If moreover $X$ is a normed linear space and also a set $V \subset X$ is given, then we say that $M$ is porous at $a$ in direction $V$ if the $b \in X$ from (i) verifying the porosity of $M$ at $a$ can be always found in the form $b=a+t v$, where $t \geq 0$ (or, equivalently, $t>0$ ) and $v \in V$. If $V=\{v\}$, then we say that $M$ is porous at $a$ in direction $v$. We say that $M$ is directionally porous at $a$ if there exists $v \in X$ such that $M$ is porous at $a$ in direction $v$.

(iii) If in the above definitions a fixed $c>0$ can be used, we speak about $c$-porosity, $c$-porosity in direction $V, c$-porosity in direction $v$ and $c$ directional porosity.

If $M \subset X$ is $c$-porous at $a \in X$ for each $0<c<1$, then we say that $M$ is strongly porous at $a$.

(iv) We say that $M$ is porous (c-porous, porous in direction $V, \ldots$ ) if $M$ is porous (c-porous, porous in direction $V, \ldots)$ at each of its points.

(v) We say that $M$ is $\sigma$-porous ( $\sigma$-c-porous, $\sigma$-porous in direction $V, \ldots)$ if it is a countable union of porous sets ( $c$-porous sets, sets porous in direction $V, \ldots)$. 
Note that $M$ is porous at $a$ in direction 0 iff $a \notin \bar{M}$; in this case $M$ is $c$-porous at $a$ in all directions for any $c>0$.

Clearly each directionally porous ( $\sigma$-directionally porous) set is porous $(\sigma$-porous) and it is an easy well-known fact that these concepts coincide in finite-dimensional spaces (cf. Lemma 4.1, (iv)).

The notion of $\sigma$-porosity was introduced by Dolzhenko [D] and since then, has been used and investigated by many authors; in some applications other variants of porosity notions are natural (cf. the survey article [Z3]).

Each $\sigma$-porous set is clearly a first category set and the Lebesgue density theorem easily implies that each $\sigma$-porous subset of $\mathbb{R}^{n}$ is of Lebesgue measure zero. Unfortunately, a $\sigma$-porous subset of an infinite-dimensional separable Banach space need not be null in any "natural measure sense". In fact, in [PT] an example of an $F_{\sigma}, \sigma$-porous subset $S$ of a separable Hilbert space $H$ is constructed in such a way that the complement $C:=H \backslash S$ intersects any line in a set of null one-dimensional Lebesgue measure (on this line); consequently $C$ is null in the Aronszajn sense and therefore it is also both null for each nondegenerate Gaussian measure on $H$ (see $[\mathrm{Ph}]$ ) and of Haar measure zero in Christensen's sense (see [Ch] for the definition). A substantial strengthening of this example has been recently found in [MM].

On the other hand, each Borel $\sigma$-directionally porous subset of a separable Banach space $X$ is null in the Aronszajn sense. This fact can be easily deduced from Aronszajn's theorem, which says that a Lipschitz function on $X$ is Gâteaux differentiable at all points except those belonging to a set which is Aronszajn null, and from the easy observation (cf. [Z2], p. 299) that a set $E \subset X$ is directionally porous at a point $a \in E$ iff the distance function $d(x):=\operatorname{dist}(x, E)$ is not Gâteaux differentiable at $a$. Nevertheless, it is interesting to try to deduce this result without any direct or indirect use of Aronszajn's Theorem. We therefore point out that an application of our decomposition theorem for $\sigma$-directionally porous sets (Theorem 4.10) supplies such an alternative argument (see Remark 4.11).

\section{Fubini Type Theorems for $\sigma$-Porous Sets: Counterex- amples}

Definition 2.1. Let $h:[0, \infty) \rightarrow[0, \infty)$ be a continuous function for which $h(0)=0$ and $h(x)>x$ for each $x>0$. We shall say that $M \subset \mathbb{R}^{2}$ is $h$-right porous at a point $c=(a, b) \in \mathbb{R}^{2}$ in direction of the $x$-axis (simply, $M$ is $(h,+)$-porous at $c)$ if for each $\varepsilon>0$ there exist $t, r>0$ such that $t<\varepsilon$, $((a+t-r, a+t+r) \times(b-r, b+r)) \cap M=\emptyset$ and $h(r)>t$.

The notion of a set which is $(h,-)$-porous at $c$ is defined in the symmetrical 
way.

We say $M \subset \mathbb{R}^{2}$ is $(h, b i l)$-porous at $c$ if $M$ is both $(h,+)$-porous and $(h,-)$-porous at $c$.

The notions of a $(h, b i l)$-porous set and a $\sigma$ - $(h, b i l)$-porous set are defined in the obvious way.

Remark 2.2. Let $h(x)=x+x^{2}$ and $M \subset \mathbb{R}^{2}$ be $(h,+)$-porous at $c$ (or $(h,-)$-porous at $c)$. Then clearly $M$ is also strongly porous at $c$.

Definition 2.3. Let $h$ be as in Definition 2.1, $G \subset \mathbb{R}^{2}$ and $\delta>0$. Then we define $W^{+}(G, h, \delta)$ as the set of all points $c=(a, b) \in \mathbb{R}^{2}$ such that there exist $0<t<\delta$ and $r>0$ such that $(a+t-r, a+t+r) \times(b-r, b+r) \subset G$ and $h(r)>t$.

The set $W^{-}(G, h, \delta)$ is defined in the obvious symmetrical way.

Remark 2.4. Obviously $M$ is $(h,+)$-porous at $x$ iff $c \in \bigcap_{k=1}^{\infty} W^{+}\left(\mathbb{R}^{2} \backslash\right.$ $\left.M, h, \frac{1}{k}\right)$. The corresponding result is true for $(h,-)$-porosity.

Lemma 2.5. Let $h$ be as in Definition 2.1, let $-\infty<t<s<\infty,-\infty<$ $v<w<\infty, 0<\eta<\frac{w-v}{2}$ and $\delta>0$ be given. Then there exists a number $t<T<s$ such that

$$
\begin{aligned}
(T, 2 s-T) & \times(v+\eta, w-\eta) \\
& \subset W^{-}((t, T) \times(v, w), h, \delta) \cap W^{+}((2 s-T, 2 s-t) \times(v, w), h, \delta) .
\end{aligned}
$$

Proof. Put $\varepsilon=\min \left(\frac{\delta}{3}, \eta, \frac{s-t}{2}\right)$. Choose $T$ such that $s-\varepsilon<T<s$ and $h(\varepsilon-(s-T))>\varepsilon+(s-T)$; this is clearly possible. Now suppose that $a \in(T, 2 s-T)$ and $b \in(v+\eta, w-\eta)$ are given. Put $p=a-(s-\varepsilon)$ and $r=\varepsilon-(s-T)$. Then clearly

$$
\begin{aligned}
& 0<p<\varepsilon+(s-T)<2 \varepsilon<\delta, \\
& h(r)>\varepsilon+(s-T)>p,
\end{aligned}
$$

and

$$
V(a-p-r, a-p+r) \times(b-r, b+r) \subset(t, T) \times(v, w) .
$$

Consequently $(a, b) \in W^{-}((t, T) \times(v, w), h, \delta)$. A quite symmetrical argument gives $(a, b) \in W^{+}((2 s-T, 2 s-t) \times(v, w), h, \delta)$.

Lemma 2.6. Let $h$ be a function as in Definition 2.1, let $\varepsilon, \delta, \omega>0$ and let $I=(a, b), K=(c, d)$ be bounded intervals. Then there exists an open interval $J \subset I$, an open set $G \subset \mathbb{R}^{2}$ and a Borel set $V \subset \mathbb{R}^{2}$ such that

(i) $\lambda\left(G_{x}\right) \leq \varepsilon$ for each $x \in \mathbb{R}$, 
(ii) $\lambda\left(V_{x}\right) \leq \omega$ for each $x \in \mathbb{R}$ and

(iii) $(J \times K) \backslash V \subset W^{-}(G, h, \delta) \cap W^{+}(G, h, \delta)$.

Proof. We may and will suppose $\omega<d-c$. Put $s=\frac{a+b}{2}$. Further choose a natural number $n$ for which $\frac{d-c}{n}<\varepsilon$ and put $\eta=\frac{\omega}{2 n}$. Now we will use Lemma $2.5 n$-times; each time with the same $h, \eta, \delta$ and $s$ but each time with different $t, v$ and $w$. In the first step we apply Lemma 2.5 to $t=t_{1}=a, v=c$ and $w=c+\frac{d-c}{n}$; we obtain the corresponding $t_{1}<T=T_{1}<s$. Then, applying the same lemma to $t_{2}=T_{1}, v=c+\frac{d-c}{n}$ and $w=c+\frac{2(d-c)}{n}$, we obtain $t_{2}<T=T_{2}<s$, we let $t_{3}=T_{2}$, and so on.

If we now put

$$
\begin{aligned}
G:= & \bigcup_{i=1}^{n}\left(\left(2 s-t_{i+1}, 2 s-t_{i}\right) \times\left(c+\frac{i-1}{n}(d-c), c+\frac{i}{n}(d-c)\right)\right. \\
& \cup \bigcup_{i=1}^{n}\left(t_{i}, t_{i+1}\right) \times\left(c+\frac{i-1}{n}(d-c), c+\frac{i}{n}(d-c)\right) \\
J:= & \left(t_{n+1}, 2 s-t_{n+1}\right)
\end{aligned}
$$

and

$$
V:=J \times\left[(c, d) \backslash \bigcup_{i=1}^{n}\left(c+\frac{i-1}{n}(d-c)+\eta, c+\frac{i}{n}(d-c)-\eta\right)\right],
$$

then conditions (i), (ii) and (iii) are clearly satisfied.

Proposition 2.7. Let $h$ be as in Definition 2.1, $K=(c, d)$ be a bounded interval and let $\xi>0$. Then there exists a Borel set $M \subset \mathbb{R} \times K$ such that

(i) $M$ is (h,bil)-porous.

(ii) There exists a residual set $A \subset \mathbb{R}$ such that $\lambda\left(M_{x}\right)>\lambda(K)-\xi$ for each $x \in A$.

Proof. Let $I_{1}, I_{2}, \ldots$ be a sequence of all intervals with rational endpoints. For each natural number $n$ we apply Lemma 2.6 to

$$
I=I_{n}, K, \delta=\frac{1}{n}, \varepsilon=\omega=\frac{\xi}{2^{n+1}} ;
$$

we obtain corresponding $J=J_{n}, G=G_{n}$ and $V=V_{n}$. Put

$$
A=\limsup _{n \rightarrow \infty} J_{n}=\bigcap_{k=1}^{\infty} \bigcup_{n=k}^{\infty} J_{n} \text { and } M=(A \times K) \backslash\left(\bigcup_{n=1}^{\infty} V_{n} \cup \bigcup_{n=1}^{\infty} G_{n}\right) .
$$


Obviously $A$ is a residual subset of $\mathbb{R}$ and $M$ is a Borel subset of $\mathbb{R}^{2}$. Since

$$
\lambda\left(M_{x}\right)>\lambda(K)-\left(\sum_{n=1}^{\infty} \lambda\left(\left(V_{n}\right)_{x}\right)+\sum_{n=1}^{\infty} \lambda\left(\left(G_{n}\right)_{x}\right) \geq \lambda(K)-\xi\right.
$$

for each $x \in A$, we have proved (ii).

If now $c \in M$ and a natural number $l$ are given, then we find $n>l$ such that $c \in\left(J_{n} \times K\right) \backslash V_{n}$. Thus by our construction (cf. Lemma 2.6,(iii)) we have

$$
c \in W^{-}\left(G_{n}, h, \frac{1}{l}\right) \cap W^{+}\left(G_{n}, h, \frac{1}{l}\right) \subset W^{-}\left(\mathbb{R}^{2} \backslash M, h, \frac{1}{l}\right) \cap W^{+}\left(\mathbb{R}^{2} \backslash M, h, \frac{1}{l}\right) .
$$

From Remark 2.4 we obtain also (i).

Theorem 2.8. Let $h$ be a function satisfying the conditions of Definition 2.1. Then there exists a Borel $\sigma-\left(h\right.$, bil)-porous set $M \subset \mathbb{R}^{2}$ such that the set $\left\{x \in \mathbb{R}: \lambda\left(\mathbb{R} \backslash M_{x}\right)=0\right\}$ is residual.

Proof. Let $K_{1}, K_{2}, \ldots$ be a sequence of all intervals with rational endpoints. By Proposition 2.7 for each $n$ there are a residual set $A_{n} \subset \mathbb{R}$ and a Borel ( $h$, bil )-porous set $M_{n} \subset \mathbb{R} \times K_{n}$ such that $\lambda\left(\left(M_{n}\right)_{x}\right)>\frac{1}{2} \lambda\left(K_{n}\right)$ for every $x \in A_{n}$. If we put $M=\bigcup_{n=1}^{\infty} M_{n}$, the Lebesgue density theorem easily implies that $\lambda\left(\mathbb{R} \backslash M_{x}\right)=0$ for each $x$ from the residual set $A:=\bigcap_{n=1}^{\infty} A_{n}$.

By Remark 2.2 we immediately obtain the following corollary.

Proposition 2.9. There exists a $\sigma$-strongly porous set $M \subset \mathbb{R}^{2}$ such that the set $\left\{x \in \mathbb{R}: \lambda\left(\mathbb{R} \backslash M_{x}\right)=0\right\}$ is residual.

The following remark concerns "very porosity" (cf. [Z3]) which is stronger than porosity and is incomparable with strong porosity.

Remark 2.10. It is easy to prove that $\left\{x \in \mathbb{R}: \lambda\left(M_{x}\right)>0\right\}$ is a first category set whenever $M \subset \mathbb{R}^{2}$ is a subset of an $F_{\sigma}$ set of Lebesgue measure zero (in particular, if $M$ is $\sigma$-very porous). Thus the analogue of Proposition 2.9 for $\sigma$-very porous sets does not hold.

\section{Directional Porosity in Products of Metric Spaces}

In the following, if $(X, \rho)$ and $(Y, \eta)$ are metric spaces, then we shall denote by $(\rho \times \eta)_{m}$ and $(\rho \times \eta)_{s}$ the maximum and the sum metric on $X \times Y$, respectively. 
Definition 3.1. Let $X, Y$ be sets and let $X \times Y$ be equipped with a metric $\omega$ and let $c>0$. Then we say that a set $A \subset X \times Y$ is $X$-directionally $c$-porous at a point $a=\left(a_{1}, a_{2}\right) \in X \times Y$ if, for each $\varepsilon>0$,

$$
\begin{aligned}
& \text { there exist } b \in X \text { and } r>0 \text { such that } \omega\left(a,\left(b, a_{2}\right)\right)<\varepsilon, \\
& r>c \omega\left(a,\left(b, a_{2}\right)\right) \text { and } B\left(\left(b, a_{2}\right), r\right) \cap A=\emptyset .
\end{aligned}
$$

The notion of a set $Y$-directionally $c$-porous at $a$ is defined in the obvious way.

For proofs we shall need the following technical notion.

Definition 3.2. Let $(X, \rho),(Y, \eta)$ be metric spaces and let $c_{x}, c_{y}>0$. Then we say that a set $A \subset X \times Y$ is $X$-directionally $\left(c_{x}, c_{y}\right)$-porous at a point $a=\left(a_{1}, a_{2}\right) \in X \times Y$ if, for each $\varepsilon>0$,

$$
\text { there exist } b \in X \text { and } r, s>0 \text { such that } \rho\left(a_{1}, b\right)<\varepsilon \text {, }
$$

$$
r>c_{x} \rho\left(a_{1}, b\right), s>c_{y} \rho\left(a_{1}, b\right) \text { and }\left(B(b, r) \times B\left(a_{2}, s\right)\right) \cap A=\emptyset .
$$

The notion of $Y$-directional $\left(c_{x}, c_{y}\right)$-porosity is defined in an obvious way.

The corresponding notions of $X$-directionally (or $Y$-directionally) $c$-porous (or $\sigma$-c-porous, or $\left(c_{1}, c_{2}\right)$-porous, or $\sigma$ - $\left(c_{1}, c_{2}\right)$-porous) sets are defined in the obvious way.

Remark 3.3. It is easy to see that the notion of $X$-directional $\left(c_{x}, c_{y}\right)$-porosity is a special case of $X$-directional $c$-porosity. In fact, it is easy to see that $A$ is $X$-directionally $\left(c_{x}, c_{y}\right)$-porous in $X \times Y$ (where $X, Y$ are equipped with metrics $\rho, \eta$, respectively) iff $A$ is $X$-directionally $c_{x}$-porous in $(X \times Y, \omega)$, where $\omega=\left(\rho \times \frac{c_{y}}{c_{x}} \eta\right)_{m}$.

To prove the existence of "small" Borel covers of "small" sets we shall need the following technical notions.

Definition 3.4. Let $(X \times Y, \omega)$ be a metric space, $A \subset X \times Y$ and let $c, \varepsilon>0$ be given. Then we define $R(A, c, \varepsilon)$ as the set of all points $a=\left(a_{1}, a_{2}\right) \in X \times Y$ for which (1) holds.

Definition 3.5. Let $(X, \rho)$ and $(Y, \eta)$ be metric spaces, $A \subset X \times Y$ and let $c_{x}, c_{y}, \varepsilon>0$ be given. Then we define $Q\left(A, c_{x}, c_{y}, \varepsilon\right)$ as the set of all points $a=\left(a_{1}, a_{2}\right) \in X \times Y$ for which (2) holds.

Lemma 3.6. Let $(X, \rho),(Y, \eta)$ be metric spaces and let $X \times Y$ be equipped with a metric $\omega$ which is (topologically) equivalent to $(\rho \times \eta)_{m}$. Let $A \subset X \times Y$ and $c, c_{x}, c_{y}, \varepsilon>0$ be given. Then each of the following holds. 
(i) $R(A, c, \varepsilon)$ and $Q\left(A, c_{x}, c_{y}, \varepsilon\right)$ are open sets in $(X \times Y, \omega)$.

(ii) $A$ is $X$-directionally c-porous at a point $a=\left(a_{1}, a_{2}\right) \in X \times Y$ iff

$$
a \in \bigcap_{\varepsilon>0} R(A, c, \varepsilon)=\bigcap_{n=1}^{\infty} R\left(A, c, \frac{1}{n}\right) .
$$

(iii) $A$ is $X$-directionally $\left(c_{x}, c_{y}\right)$-porous at a point $a=\left(a_{1}, a_{2}\right) \in X \times Y$ iff

$$
a \in \bigcap_{\varepsilon>0} Q\left(A, c_{x}, c_{y}, \varepsilon\right)=\bigcap_{n=1}^{\infty} Q\left(A, c_{x}, c_{y}, \frac{1}{n}\right) .
$$

(iv) If $A$ is $X$-directionally c-porous $\left(\left(c_{x}, c_{y}\right)\right.$-porous), then there exists a $G_{\delta}$ set $\tilde{A} \supset A\left(A^{*} \supset A\right)$ which is $X$-directionally c-porous ( $\left(c_{x}, c_{y}\right)$-porous).

(v) If $A$ is $\sigma$-X-directionally c-porous $\left(\left(c_{x}, c_{y}\right)\right.$-porous $)$, then there exists a $G_{\delta \sigma}$ set $\tilde{A} \supset A\left(A^{*} \supset A\right)$ which is $\sigma$-X-directionally c-porous $\left(\left(c_{x}, c_{y}\right)\right.$ porous).

Proof. The statements (i), (ii) and (iii) are obvious. If $A$ is $X$-directionally $c$-porous, we put $\tilde{A}=\bar{A} \cap \bigcap_{n=1}^{\infty} R(A, c, 1 / n)$. By (i), $\tilde{A}$ is a $G_{\delta}$ set. By (ii) and by the obvious fact, that if $A$ is $X$-directionally $c$-porous at a point $a$, then any subset of $\bar{A}$ is $X$-directionally $c$-porous at $a$, we obtain that $A \subset \tilde{A}$ and $\tilde{A}$ is $X$-directionally $c$-porous. This proves the first part of (iv). The second part is an immediate consequence of the first one and Remark 3.3. The assertion (v) follows easily by (iv).

Lemma 3.7. Let $(X, \rho),(Y, \eta)$ be metric spaces, let $X \times Y$ be equipped with the sum metric $\omega=(\rho \times \eta)_{s}$, and let $0<\alpha<\frac{1}{2}$ be given. Then each of the following holds.

(i) If a set $A \subset X \times Y$ is $(1-\alpha)$-porous at a point $a=\left(a_{1}, a_{2}\right) \in X \times Y$, then either $A$ is $X$-directionally $(1-2 \alpha)$-porous at a or $A$ is $Y$-directionally $(1-2 \alpha)$-porous at a.

(ii) If $A$ is $(1-\alpha)$-porous, then we can write $A=A_{1} \cup A_{2}$, where $A_{1}$ is $X$ directionally $(1-2 \alpha)$-porous and $A_{2}$ is $Y$-directionally $(1-2 \alpha)$-porous.

Proof. Suppose that the assertion of (i) is not true. Then there exists $\varepsilon>0$ such that

$$
r_{1} \leq(1-2 \alpha) \rho\left(a_{1}, x_{1}\right) \text { if } B\left(\left(x_{1}, a_{2}\right), r_{1}\right) \cap A=\emptyset \text { and } \rho\left(a_{1}, x_{1}\right)<\varepsilon
$$


and

$$
r_{2} \leq(1-2 \alpha) \eta\left(a_{2}, x_{2}\right) \text { if } B\left(\left(a_{1}, x_{2}\right), r_{2}\right) \cap A=\emptyset \text { and } \eta\left(a_{2}, x_{2}\right)<\varepsilon \text {. }
$$

By our assumption we can find a point $x=\left(x_{1}, x_{2}\right)$ and $r>0$ such that $\omega(a, x)<\varepsilon, B(x, r) \cap A=\emptyset$ and

$$
r>(1-\alpha) \omega(a, x)=(1-\alpha)\left(\rho\left(a_{1}, x_{1}\right)+\eta\left(a_{2}, x_{2}\right)\right) .
$$

Put $r_{1}=r-\eta\left(a_{2}, x_{2}\right)$ and $r_{2}=r-\rho\left(a_{1}, x_{1}\right)$. Clearly we have (if $r_{1}>0$ ) $B\left(\left(x_{1}, a_{2}\right), r_{1}\right) \subset B(x, r) \subset(X \times Y) \backslash A$ and $\rho\left(a_{1}, x_{1}\right)<\varepsilon$. Thus (3) implies

$$
r-\eta\left(a_{2}, x_{2}\right) \leq(1-2 \alpha) \rho\left(a_{1}, x_{1}\right)
$$

By a symmetrical argument, (4) implies

$$
r-\rho\left(a_{1}, x_{1}\right) \leq(1-2 \alpha) \eta\left(a_{2}, x_{2}\right)
$$

Adding the last two inequalities, we obtain

$$
r \leq(1-\alpha)\left(\rho\left(a_{1}, x_{1}\right)+\eta\left(a_{2}, x_{2}\right)\right)
$$

which contradicts (5). Thus (i) is proved.

To prove (ii), it is clearly sufficient to define $A_{1}\left(A_{2}\right)$ as the set of points in $A$ at which $A$ is $X$-directionally ( $Y$-directionally) $(1-2 \alpha)$-porous.

Using the above lemma and Theorem 4.5 of [Z1] according to which, for any $0<c<1$, any $\sigma$-porous subset of a metric space is $\sigma$-c-porous, we easily obtain the following decomposition theorem.

Theorem 3.8. Let $(X, \rho),(Y, \eta)$ be metric spaces and let $X \times Y$ be equipped with the sum metric. Let $A \subset X \times Y$ be a $\sigma$-porous set and let $0<c<1$ be given. Then we can write $A=A_{1} \cup A_{2}$, where $A_{1}$ is $\sigma$-X-directionally c-porous and $A_{2}$ is $\sigma-Y$-directionally c-porous.

Proof. Find $\varepsilon>0$ such that $c<1-2 \varepsilon$. By Theorem 4.5 of [Z1], $A$ is $\sigma-(1-\varepsilon)-$ porous set. Using this fact and Lemma 3.7,(ii), we obtain our assertion.

Remark 3.9. (i) If $A$ is Borel, then the sets $A_{1}, A_{2}$ in Theorem 3.8 can be chosen to be Borel. In fact, we can write $A=\left(A \cap \tilde{A}_{1}\right) \cup\left(A \cap \tilde{A_{2}}\right)$, where $\tilde{A}_{1}, \tilde{A}_{2}$ are $G_{\delta \sigma}$ covers from Lemma 3.6,(v).

(ii) Of course, $A_{1}\left(A_{2}\right)$ has the property that all sections $\left(A_{1}\right)^{y}\left(\left(A_{2}\right)_{x}\right)$ are $\sigma$-porous subsets of $X(Y)$. 
If $P$ is a metric space and $\mu$ is a measure on $P$, we say that $\mu$ is absolutely continuous w.r.t. $\sigma$-porous sets if $\mu(M)=0$ for each $\sigma$-porous set $M \subset P$. Note that every Radon measure on $\mathbb{R}$ which is absolutely continuous w.r.t. Lebesgue measure is clearly absolutely continuous w.r.t. $\sigma$-porous sets but the opposite implication does not hold (see $[\mathrm{T}]$ ).

Remark 3.9 easily implies the following proposition which seems to be of some independent interest and which suggests that Theorem 3.8 is in fact a substitute for the (invalid) "Fubini type" theorem for $\sigma$-porous sets.

Proposition 3.10. Let $\mu$ and $\nu$ be Radon measures on separable metric spaces $X$ and $Y$, respectively, which are absolutely continuous w.r.t. $\sigma$-porous sets. Then the Radon product measure $\mu \otimes \nu$ is absolutely continuous w.r.t. $\sigma$-porous sets as well.

If we use Theorem 3.8 in $\mathbb{R}^{2}$ equipped with the Euclidean norm, we easily obtain the following result, in which we put $e_{1}=(1,0)$ and $e_{2}=(0,1)$.

Proposition 3.11. Let $A \subset \mathbb{R}^{2}$ be a $\sigma$-porous set and let $0<c<\frac{1}{\sqrt{2}}$. Then

$$
A=A_{1}^{+} \cup A_{1}^{-} \cup A_{2}^{+} \cup A_{2}^{-},
$$

where $A_{i}^{+}\left(A_{i}^{-}\right)$is a set which is $\sigma$-c-porous in direction $e_{i}\left(-e_{i}\right), i=1,2$.

Remark 3.12. If we consider $A=\{(x, x): x \in \mathbb{R}\}$, we easily observe (using the Baire category theorem) that the assertion of Proposition 3.11 does not hold for $c=\frac{1}{\sqrt{2}}$.

Remark 3.13. Lemma 3.7 and subsequently also Theorem 3.8 can be easily generalized to the case of the product of several metric spaces. Further we can easily generalize Proposition 3.11 to $\mathbb{R}^{n}$ (with the assumption $0<c<\frac{1}{\sqrt{n}}$ ).

In the proof of Theorem 3.8 we applied the method of "enlargement of the porosity index" established in Proposition 4.1 from [Z1] (which implies Theorem 4.5 from [Z1]) to the sum metric in the product of two metric spaces. In the following lemma and its consequence Proposition 3.15 we now show how this method may be generalized in the special situation of product spaces and directional porosity. These results will be used in the following section (see (4.12)-(4.15)).

Lemma 3.14. Let $(X, \rho)$ and $(Y, \eta)$ be metric spaces, $1>c_{x}>0, c_{y}>0$ and let $A \subset X \times Y$ be an $X$-directionally $\left(c_{x}, c_{y}\right)$-porous set. Then $A$ is $\sigma$-Xdirectionally $\left(\sqrt{c_{x}}, \frac{c_{y}}{2}\right)$-porous set. 
Proof. Put $A_{0}=A \cap \bigcap_{n=1}^{\infty} Q\left(A, \sqrt{c_{x}}, \frac{c_{y}}{2}, \frac{1}{n}\right)$. By Lemma 3.6,(iii) we have that $A_{0}$ is an $X$-directionally $\left(\sqrt{c_{x}}, \frac{c_{y}}{2}\right)$-porous set. Since $A \backslash A_{0}=\bigcup_{n=1}^{\infty}(A \backslash$ $\left.Q\left(A, \sqrt{c_{x}}, \frac{c_{y}}{2}, \frac{1}{n}\right)\right)$, it is sufficient to prove that each of the sets $A_{n}:=A \backslash$ $Q\left(A, \sqrt{c_{x}}, \frac{c_{y}}{2}, \frac{1}{n}\right)$ is $X$-directionally $\left(\sqrt{c_{x}}, \frac{c_{y}}{2}\right)$-porous. To this end choose an arbitrary natural number $n, 0<\varepsilon<\frac{1}{n}$ and $(x, y) \in A_{n}$. It is sufficient to prove that

$$
(x, y) \in Q\left(A_{n}, \sqrt{c_{x}}, \frac{c_{y}}{2}, \varepsilon\right) .
$$

Since $(x, y) \in A$, we know that $(x, y) \in Q\left(A, c_{x}, c_{y}, \varepsilon\right)$ and therefore we can choose $b \in X$ and $r, s>0$ such that $\left.\rho(x, b)<\varepsilon, r>c_{x} \rho(x, b), s>c_{y} \rho_{(} x, b\right)$ and $(B(b, r) \times B(y, s)) \cap A=\emptyset$. Since $r>c_{x} \rho(x, b)$, we can choose $r^{*}>0$ such that $\sqrt{c_{x}} \rho(x, b)<r^{*}<\rho(x, b)$ and $r>\sqrt{c_{x}} r^{*}$. To prove (6), it is clearly sufficient to prove that $A_{n} \cap\left(B\left(b, r^{*}\right) \times B\left(y, \frac{s}{2}\right)\right)=\emptyset$. Assuming that this is not the case, we choose an $\left(x_{1}, y_{1}\right) \in A_{n} \cap\left(B\left(b, r^{*}\right) \times B\left(y, \frac{s}{2}\right)\right)$. Clearly,

$$
B(b, r) \times B\left(y_{1}, \frac{s}{2}\right) \subset B(b, r) \times B(y, s) \subset(X \times Y) \backslash A .
$$

Since

and

$$
\begin{aligned}
& \rho\left(x_{1}, b\right)<r^{*}<\rho(x, b)<\varepsilon<\frac{1}{n}, \\
& r>\sqrt{c_{x}} r^{*}>\sqrt{c_{x}} \rho\left(x_{1}, b\right)
\end{aligned}
$$

$$
\frac{s}{2}>\frac{c_{y}}{2} \rho(x, b)>\frac{c_{y}}{2} r^{*}>\frac{c_{y}}{2} \rho\left(x_{1}, b\right),
$$

we conclude that $\left(x_{1}, y_{1}\right) \in Q\left(A, \sqrt{c_{x}}, \frac{c_{y}}{2}, \frac{1}{n}\right)$, which contradicts the fact that $\left(x_{1}, y_{1}\right) \in A_{n}$.

Proposition 3.15. Let $(X, \rho),(Y, \eta)$ be metric spaces, let $c_{x}, c_{y}>0,0<\alpha<$ 1 , and let $A \subset X \times Y$ be a $\sigma$-X-directionally $\left(c_{x}, c_{y}\right)$-porous set. Then there exists $\beta>0$ such that $A$ is $\sigma$-X-directionally $(\alpha, \beta)$-porous.

Proof. We may suppose $c_{x}<1$. Choose a natural number $n$ so large that $\left(c_{x}\right)^{2^{-n}}>\alpha$ and put $\beta=2^{-n} c_{y}$. Then, applying Lemma $3.14 n$-times, we obtain that $A$ is $\sigma$-X-directionally $(\alpha, \beta)$-porous.

\section{Sigma-Directionally Porous Sets in Banach Spaces}

We start with the following easy properties of directional porosity.

Lemma 4.1. Let $X$ be a normed linear space, $M \subset X, V \subset X, W \subset X, a \in X$ and $c>0$. Then the following assertions hold. 
(i) If $0 \notin V$, then $M$ is porous (c-porous) at a in direction $V$ iff $M$ is porous (c-porous) at a in direction $V_{n}$, where $V_{n}=\left\{\frac{v}{\|v\|}: v \in V\right\} . M$ is porous (c-porous) at a in direction $v$ iff it is porous (c-porous) at a in direction $\{t v: t \geq 0\}$.

(ii) If $V=V_{1} \cup V_{2} \cup \ldots \cup V_{n}$ and $M$ is c-porous at a in direction $V$, then there exists $1 \leq i \leq n$ such that $M$ is c-porous at a in direction $V_{i}$. If $M$ is porous (c-porous) in direction $V$, then we can write $M=\bigcup_{i=1}^{n} M_{i}$, where $M_{i}$ is porous (c-porous) in direction $V_{i}$.

(iii) Let $V, W \subset\{x \in X:\|x\|=1\}, 0<\omega<c$ and let dist $(v, W)<c-\omega$ for each $v \in V$. Let $M$ be c-porous at a in direction $V$. Then $M$ is $\omega$-porous at a in direction $W$.

(iv) If $V$ is a compact subset of $\{x \in X:\|x\|=1\}$, then $M$ is porous at a in direction $V$ iff there exists $v \in V$ such that $M$ is porous at a in direction $v$. In particular, if $X$ is a finite-dimensional space, then $M$ is porous at a iff $M$ is directionally porous at a.

Proof. The statement (i) and the first part of (ii) are obvious. To prove the second part of (ii) it is sufficient to define $M_{i}$ as the set of points in $M$ at which $M$ is porous (c-porous) in direction $V_{i}$.

To prove (iii), choose an arbitrary $\varepsilon>0$. Since $M$ is $c$-porous at $a$ in direction $V$, there exist $t \geq 0, v \in V$ and $r>0$ such that $B(a+t v, r) \cap M=$ $\emptyset$, $\|t v\|=t<\varepsilon$ and $r>c\|t v\|=c t$. Find $w \in W$ such that $\|v-w\|<c-\omega$ and put $b^{*}=a+t w, r^{*}=r-t\|v-w\|$. Then $\left\|b^{*}-a\right\|=\|t w\|=t<$ $\varepsilon, \omega\|t w\|=\omega t=c t-(c-\omega) t<r-t\|v-w\|=r^{*}$ and $B\left(b^{*}, r^{*}\right) \cap M=\emptyset$, since clearly $B\left(b^{*}, r^{*}\right) \subset B(a+t v, r)$. Thus $M$ is $\omega$-porous at $a$ in direction $W$.

To prove (iv) suppose that $V \subset\{x \in X:\|x\|=1\}$ is a compact set and $M$ is $c$-porous at $a$ in direction $V$. Find points $w_{1}, w_{2}, \ldots, w_{n}$ from $V$ such that dist $(v, W)<\frac{c}{3}$ for each $v \in V$, where $W=\left\{w_{1}, \ldots, w_{n}\right\}$. By (iii) $M$ is $\frac{c}{3}$-porous at $a$ in direction $W$; thus by (ii) $M$ is directionally porous at $a$.

The following notation is useful.

Definition 4.2. Let $X$ be a normed linear space, $A \subset X, Z \subset X, c>0$ and $\varepsilon>0$. Then we denote by $P(A, Z, c, \varepsilon)$ the set of all points $a \in X$ for which there exist $z \in Z, t \geq 0$ and $r>0$ such that $B(a+t z, r) \cap A=\emptyset,\|t z\|<\varepsilon$ and $r>c\|t z\|$.

Lemma 4.3. Let $X$ be a normed linear space, $A \subset X, Z \subset X, a \in X, c>0$ and $\varepsilon>0$. Then the following assertions are true. 
(i) $P(A, Z, c, \varepsilon)$ is an open set.

(ii) $A$ is c-porous at a in direction $Z$ iff

$$
a \in \bigcap_{\varepsilon>0} P(A, Z, c, \varepsilon)=\bigcap_{n=1}^{\infty} P\left(A, Z, c, \frac{1}{n}\right) .
$$

(iii) $A$ is porous at a in direction $Z$ iff

$$
a \in \bigcup_{k=1}^{\infty} \bigcap_{n=1}^{\infty} P\left(A, Z, \frac{1}{k}, \frac{1}{n}\right) .
$$

(iv) If $A$ is c-porous in direction $Z$, then there exists a $G_{\delta}$-set $\tilde{A} \supset A$ which is c-porous in direction $Z$.

(v) If $A$ is porous ( $\sigma$-porous) in direction $Z$, then there exists a $G_{\delta \sigma}$-set $A^{*} \supset A$ which is porous ( $\sigma$-porous) in direction $Z$.

(vi) If $A$ is porous in direction $Z$ (directionally porous), then we can write $A=\bigcup_{k=1}^{\infty} A_{k}$, where each $A_{k}$ is $\frac{1}{k}$-porous in direction $Z$ ( $\frac{1}{k}$-directionally porous).

Proof. The statements (i), (ii) and (iii) are obvious.

If $A$ is $c$-porous in direction $Z$, we put $\tilde{A}=\bar{A} \cap \bigcap_{n=1}^{\infty} P\left(A, Z, c, \frac{1}{n}\right)$. If $A$ is porous in direction $Z$, then we put

$$
A^{*}:=\bar{A} \cap \bigcup_{k=1}^{\infty} \bigcap_{n=1}^{\infty} P\left(A, Z, \frac{1}{k}, \frac{1}{n}\right) .
$$

By (i) $\tilde{A}$ is a $G_{\delta}$ set and $A^{*}$ is a $G_{\delta \sigma}$-set. By (ii), (iii) and by the obvious fact, that if $A$ is porous (c-porous) at a point $a$ in direction $Z$, then any subset of $\bar{A}$ is porous (c-porous) at $a$ in direction $Z$, we obtain that $\tilde{A} \supset A, A^{*} \supset A, \tilde{A}$ is $c$-porous in direction $Z$ and $A^{*}$ is porous in direction $Z$. This proves (iv) and the statement of $(\mathrm{v})$ concerning porosity; it then easily implies the second statement of (v) concerning $\sigma$-porosity. To prove (vi), it is clearly sufficient to define $A_{k}$ as the set of all points in $A$ at which $A$ is $\frac{1}{k}$-porous in direction $Z$ $\left(\frac{1}{k}\right.$-directionally porous).

For the brevity of formulations, we now define an auxiliary notion of admissible pair of sets.

Definition 4.4. Let $X$ be a normed linear space and let $E, F \subset X$ be given. We say that $E, F$ is an admissible pair, if there exists $\eta>0$ such that

$$
\|e+f\| \geq \eta \max (\|e\|,\|f\|) \text { whenever } e \in E, f \in F \text {. }
$$


Remark 4.5. Obviously, if there exist two topologically complementary closed spaces $V_{1}, V_{2} \subset X$ such that $E \subset V_{1}$ and $F \subset V_{2}$, then $E, F$ is an admissible pair.

Lemma 4.6. Let $X$ be a normed linear space, $E, F \subset X$ be an admissible pair, $c>0$ and let $A \subset X$ be c-porous in direction $E+F$. Then $A$ can be written in the form $A=A_{1} \cup A_{2}$, where $A_{1}$ is porous in direction $E$ and $A_{2}$ is $\sigma$-porous in direction $F$.

Proof. Let $\eta>0$ be a number corresponding to $E, F$ from Definition 4.4 and put $A_{1}=A \cap \bigcap_{m=1}^{\infty} P\left(A, E, \frac{c \eta}{2}, \frac{1}{m}\right)$. By Lemma 4.3 ,(ii) we obtain that $A_{1}$ is $\frac{c \eta}{2}$-porous in direction $E$. Thus it is sufficient to prove that

$$
A \backslash A_{1}=\bigcup_{m=1}^{\infty}\left(A \backslash P\left(A, E, \frac{c \eta}{2}, \frac{1}{m}\right)\right) \text { is } \sigma \text {-porous in direction } F \text {. }
$$

To this end we shall show that each set $D_{m}:=A \backslash P\left(A, E, \frac{c \eta}{2}, \frac{1}{m}\right)$ is $\frac{c \eta}{2}$-porous in direction $F$. To prove this, choose arbitrary $x \in D_{m}$ and $0<\varepsilon<\frac{1}{m}$. We need to prove that

$$
x \in P\left(D_{m}, F, \frac{c \eta}{2}, \varepsilon\right) .
$$

Since $x \in A$, we know that $A$ is $c$-porous at $x$ in direction $E+F$ and consequently we can find $e \in E, f \in F, t>0$ and $r>0$ such that

$$
B(x+t(e+f), r) \cap A=\emptyset,\|t(e+f)\|<\varepsilon \eta \text { and } r>c\|t(e+f)\| .
$$

By the definition of $\eta$,

$$
\max (\|t e\|,\|t f\|) \leq \frac{1}{\eta}\|t e+t f\|<\varepsilon<\frac{1}{m} .
$$

To prove (7), it is sufficient to show that $B\left(x+t f, \frac{r}{2}\right) \cap D_{m}=\emptyset$, since (8) implies $\|t f\|<\varepsilon$ and also $\frac{r}{2}>\frac{c}{2}\|t(e+f)\| \geq \frac{c \eta}{2}\|t f\|$. Thus suppose to the contrary that there is a $y \in B\left(x+t f, \frac{r}{2}\right) \cap D_{m}$. Clearly $B\left(y+t e, \frac{r}{2}\right) \subset B(x+t(e+f), r)$ and therefore $B\left(y+t e, \frac{r}{2}\right) \cap A=\emptyset$. Since (8) implies $\|t e\|<\frac{1}{m}$ and

$$
\frac{r}{2}>\frac{c}{2}\|t(e+f)\| \geq \frac{c \eta}{2}\|t e\|
$$

we obtain that $y \in P\left(A, E, \frac{c \eta}{2}, \frac{1}{m}\right)$, which contradicts the fact that $y \in D_{m}$.

Lemma 4.6 and Lemma 4.3,(vi) clearly imply the following proposition. 
Proposition 4.7. Let $X$ be a normed linear space and let $E, F \subset X$ be an admissible pair. Then each $A \subset X$ which is $\sigma$-porous in direction $E+F$ can be written in the form $A=A_{1} \cup A_{2}$, where $A_{1}$ is $\sigma$-porous in direction $E$ and $A_{2}$ is $\sigma$-porous in direction $F$.

Proposition 4.8. Let $X$ be a normed linear space and let its finite dimensional subspace $V$ be spanned by $v_{1}, \ldots, v_{n}$. Let $A \subset X$ be $\sigma$-porous in direction $V$. Then we can write $A=\bigcup_{k=1}^{n}\left(A_{k}^{+} \cup A_{k}^{-}\right)$, where $A_{k}^{+}, A_{k}^{-}$are sets $\sigma$-porous in directions $v_{k},-v_{k}$, respectively.

Proof. We shall proceed by induction. For $n=1$ we infer the statement from Lemma 4.1, (i) and (ii).

Assume now that $n>1$ and the statement holds with $n$ replaced by $n-1$. Suppose that $V, v_{1}, \ldots, v_{n}$, and $A$ are as in the assumptions of the proposition, and that, without loss of generality, $v_{1}, \ldots, v_{n}$ are linearly independent. Since $V=E+F$, where $E=\operatorname{span}\left\{v_{1}, \ldots, v_{n-1}\right\}$ and $F=\operatorname{span}\left\{v_{n}\right\}$, and the pair $E, F$ is clearly admissible, Proposition 4.7 and the induction assumption easily imply the assertion.

Now we are ready to deduce the announced decomposition results for directionally porous sets.

Proposition 4.9. Let $V=\overline{\operatorname{span}\left\{v_{1}, v_{2}, \ldots\right\}}$ be a subspace of a normed linear space $X$. Let $A \subset X$ be a set such that for each point $a \in A$ there exists $v_{a} \in V$ such that $A$ is porous at a in direction $v_{a}$. Then we can write $A=\bigcup_{n=1}^{\infty}\left(A_{n}^{+} \cup\right.$ $\left.A_{n}^{-}\right)$, where $A_{n}^{+}, A_{n}^{-}$are sets $\sigma$-porous in directions $v_{n},-v_{n}$, respectively.

Proof. We may and will suppose $\left\|v_{a}\right\|=1$. Let $A_{k, n}$ be the set of all points $a \in A$, at which $A$ is $\frac{1}{k}$-porous in direction $v_{a}$ and

$$
\operatorname{dist}\left(v_{a}, \operatorname{span}\left\{v_{1}, \ldots, v_{n}\right\} \cap\{v \in X:\|v\|=1\}\right)<\frac{1}{2 k} .
$$

It is easy to see that $A=\bigcup_{k, n=1}^{\infty} A_{k, n}$. Lemma 4.1, (iii) (applied to $V:=\left\{v_{a}\right\}$, $\left.W:=\operatorname{span}\left\{v_{1}, \ldots, v_{n}\right\} \cap\{v \in X:\|v\|=1\}, c:=\frac{1}{k}, \omega:=\frac{1}{2 k}\right)$ easily implies that $A_{k, n}$ is porous in direction span $\left\{v_{1}, \ldots, v_{n}\right\}$. Thus Proposition 4.8 immediately gives our assertion.

The following theorem captures the most interesting special case of Proposition 4.9 .

Theorem 4.10. Let $X$ be a separable normed linear space and let $\left(v_{n}\right)_{1}^{\infty}$ be a complete sequence in $X$ (i.e., $\overline{\operatorname{span}\left\{v_{1}, \ldots\right\}}=X$ ). Let $A \subset X$ be a $\sigma$-directionally porous set. Then we can write $A=\bigcup_{n=1}^{\infty}\left(A_{n}^{+} \cup A_{n}^{-}\right)$, where $A_{n}^{+}, A_{n}^{-}$are sets $\sigma$-porous in directions $v_{n},-v_{n}$, respectively. 
Remark 4.11. We can demand in Proposition 4.9 and Theorem 4.10 that the sets $A_{n}^{+}, A_{n}^{-}$are Borel if $A$ is Borel. In fact, on account of Lemma 4.3,(v) we see that they can be replaced by sets $\left(A_{n}^{+}\right)^{*} \cap A$ and $\left(A_{n}^{-}\right)^{*} \cap A$. This observation immediately implies that each Borel $\sigma$-directionally porous subset of a separable Banach space is null in the Aronszajn sense - the fact which was deduced in Introduction from Aronszajn's differentiability theorem.

Remark 4.12. Our method of proof of the decomposition results, based on Lemma 4.6, works also in cases when we want to distinguish between the "onesided" and the "bilateral" directional porosity in Banach spaces; in such cases we may apply Proposition 4.7.

In certain other circumstances, it is possible to use Proposition 3.15 instead of Lemma 4.6. For example, Proposition 4.8 may be easily obtained by using Proposition 3.15 in $\left(X_{1} \times \cdots \times X_{n}\right) \times Y$, where $X_{1} \times \cdots \times X_{n}$ is equipped with the sum metric, and a generalization of Lemma 3.7. This provides an alternative approach to the proof of Theorem 4.10.

We will now strengthen Theorem 4.10 by showing that the sets $A_{n}^{+}, A_{n}^{-}$can be chosen to be even "more $\sigma$-porous" in the sense of the following definition. This application of Proposition 3.15 is different from that indicated in the preceding remark, since our arguments use Proposition 3.15 together with Theorem 4.10.

Definition 4.13. Let $X$ be a normed linear space and let $a, v \in X$. If $c, \gamma>0$ and $A \subset X$ are given, then we say that $A$ is $[c, \gamma]$-porous at $a$ in direction $v$ if for each $\varepsilon>0$ there exist $t \geq 0$ and $r, s>0$ such that $\|t v\|\langle\varepsilon, r>c t, s>\gamma\|t v\|$ and

$$
B(a+(t+\tau) v, s) \cap A=\emptyset \text { for each }-r \leq \tau \leq r .
$$

We say that $A$ is $[c, \gamma]$-porous in direction $v$ if $A$ is $[c, \gamma]$-porous at each of its points.

Theorem 4.14. Let $X$ be a separable normed linear space, $0<c<1$ and let $\left(v_{n}\right)_{1}^{\infty}$ be a complete sequence in $X$ (i.e., $\overline{\operatorname{span}\left\{v_{1}, \ldots\right\}}=X$ ). Let $A \subset X$ be a $\sigma$-directionally porous set. Then we can write $A=\bigcup_{k=1}^{\infty} C_{k}$, where for each $k$ there exist $\gamma_{k}>0$, an index $n$ and $v^{k} \in X$ such that $v^{k} \in\left\{v_{n},-v_{n}\right\}$ and the set $C_{k}$ is $\left[c, \gamma_{k}\right]$-porous in direction $v^{k}$.

Proof. We may assume that $v_{n} \neq 0$ for each $n$. On account of Theorem 4.10 and Lemma 4.3,(vi) we may suppose that $A$ is $c$-porous in direction $v$, where $c>0$ and $v=v_{n}$ or $v=-v_{n}$ for some $n$. Let $P$ be a norm one projection of $X$ onto $V:=\operatorname{span}\{v\}$, and let $W$ be the kernel of $P$. 
Put $\alpha=\frac{c+1}{2}=c+\frac{1-c}{2}$. For any $a=a_{1}+a_{2}$, where $a_{1} \in V$ and $a_{2} \in W$, and for any $r>0$ the ball $B(a, r)$ contains the sum of balls $B_{V}\left(a_{1}, r / 2\right)$ and $B_{W}\left(a_{2}, r / 2\right)$, where the symbols $B_{V}$ and $B_{W}$ are used for balls in $V$ and $W$, respectively. Thus we infer that, in the natural identification of $X=V \oplus W$ with $V \times W$, the set $A$ is $V$-directionally $(c / 2, c / 2)$-porous, and we conclude from Proposition 3.15 that there is $\beta>0$ such that $A=\bigcup_{n=1}^{\infty} A_{n}$ for some $V$-directionally $(\alpha, \beta)$-porous sets $A_{n}$.

Fix an index $n$ and choose arbitrary $a \in A_{n}$ and $\varepsilon>0$. Let $a=a_{1}+a_{2}$, where $a_{1} \in V$ and $a_{2} \in W$. Since $A_{n}$ is $V$-directionally $(\alpha, \beta)$-porous, we can find $b \in V, r^{*}>\alpha\left\|b-a_{1}\right\|$ and $s^{*}>\beta\left\|b-a_{1}\right\|$ such that $\left\|b-a_{1}\right\|<\varepsilon$ and $\left(B_{V}\left(b, r^{*}\right) \times B_{W}\left(a_{2}, s^{*}\right)\right) \cap A_{n}=\emptyset$.

Now let $t \neq 0$ be such that $b-a_{1}=t v$. Further put

$$
\gamma=\min \left(\frac{1-c}{8}, \frac{\beta}{4}\right), r=\left(\frac{3 c+1}{4}\right)|t| \text { and } s=2 \gamma\|t v\| .
$$

Then clearly $\|t v\|<\varepsilon, r>c|t|$ and $s>\gamma\|t v\| ;$ so, to finish the proof, it suffices to show that $B(a+(t+\tau) v, s) \subset B_{V}\left(b, r^{*}\right)+B_{W}\left(a_{2}, s^{*}\right)$ whenever $-r \leq \tau \leq r$. But this is clear, because for any $-r \leq \tau \leq r$ and $z \in B(a+(t+\tau) v, s)$ we have that $z=P(z)+(z-P(z))$, where $P(z) \in V, z-P(z) \in W, P(z) \in B_{V}\left(b, r^{*}\right)$ since

$\|P(z)-b\| \leq\|\tau v\|+\|z-(a+(t+\tau) v)\|<\left(\frac{3 c+1}{4}+2 \gamma\right)\|t v\| \leq \alpha\left\|b-a_{1}\right\|<r^{*}$

and $z-P(z) \in B_{W}\left(a_{2}, s^{*}\right)$ since

$$
\begin{aligned}
\left\|z-P(z)-a_{2}\right\| & =\|(z-(a+(t+\tau) v))-P(z-(a+(t+\tau) v))\| \\
& <2 s \leq \beta\left\|b-a_{1}\right\|<s^{*} .
\end{aligned}
$$

Remark 4.15. For convenience, we have stated the strengthened version of Theorem 4.10 only. The above proof, however, gives also the corresponding strengthening of Proposition 4.9.

\section{References}

[A] N. Aronszajn, Differentiability of Lipschitzian mappings between Banach spaces, Studia Math. 57 (1976), 147-190.

[Ch] J.P.R. Christensen, Topology and Borel structure, North-Holland, Amsterdam 1974.

[D] E. P. Dolzhenko, Boundary properties of arbitrary functions (in Russian), Izv. Akad. Nauk SSSR Ser. Mat. 31 (1967), 3-14. 
[F] J.Foran, Continuous functions need not have $\sigma$-porous graphs, Real Analysis Exchange 11 (1985-86), 194-203.

[MM] J. Matoušek and E. Matoušková, A highly nonsmooth norm on Hilbert space, Israel J. Math., to appear.

[O] J. C. Oxtoby, Measure and Category, (2 nd edition), Springer-Verlag, New York Heidelberg Berlin, 1980.

[Ph] R. R. Phelps, Gaussian null sets and differentiability of Lipschitz maps on Banach spaces, Pacific J. Math. 77 (1978), 523-531.

[PT] D. Preiss and J. Tišer, Two unexpected examples concerning differentiability of Lipschitz functions on Banach spaces, in Collection: Geometric aspects of functional analysis, 219-238, Oper. Theory Adv. Appl. 77, Birkhauser 1995.

[PZ] D. Preiss and L. Zajíček, On ordinary and Michel-Penot upper directional derivatives of Lipschitz functions, in preparation.

[T] J. Tkadlec, Construction of a finite Borel measure with $\sigma$-porous sets as null sets, Real Analysis Exchange 12 (1986-87), 349-353.

[Z1] L. Zajičček, Sets of ( $\sigma)$-porosity and sets of $(\sigma)$-porosity ( $q$ ), Časopis Pěst. Mat. 101 (1976), 350-359.

[Z2] L. Zajíček, Differentiability of of the distance function and points of multi-valuedness of the metric projection in Banach space, Czechoslovak Math. J. 33 (108) (1983), 292-308.

[Z3] L. Zajíček, Porosity and $\sigma$-porosity, Real Analysis Exchange 13 (198788), 314-350.

[Z4] L. Zajíček, Products of non- $\sigma$-porous sets and Foran systems, Atti Sem. Mat. Fis. Univ. Modena. 44 (1996), 497-505. 
D. Preiss L. ZAJÍČEK 